Pacific

Journal of

Mathematics

LOCAL MOVES ON SPATIAL GRAPHS

AND FINITE TYPE INVARIANTS

Kouki Taniyama And Akira Yasuhara 


\title{
LOCAL MOVES ON SPATIAL GRAPHS AND FINITE TYPE INVARIANTS
}

\author{
KOUKI TANIYAMA AND AKIRA YASUHARA
}

\begin{abstract}
We define $A_{k}$-moves for embeddings of a finite graph into the 3-sphere for each natural number $k$. Let $A_{k}$-equivalence denote an equivalence relation generated by $A_{k}$-moves and ambient isotopy. $A_{k}$-equivalence implies $A_{k-1}$-equivalence. Let $\mathcal{F}$ be an $A_{k-1}$-equivalence class of the embeddings of a finite graph into the 3 -sphere. Let $\mathcal{G}$ be the quotient set of $\mathcal{F}$ under $A_{k}$-equivalence. We show that the set $\mathcal{G}$ forms an abelian group under a certain geometric operation. We define finite type invariants on $\mathcal{F}$ of order $(n ; k)$. And we show that if any finite type invariant of order $(1 ; k)$ takes the same value on two elements of $\mathcal{F}$, then they are $A_{k}$-equivalent. $A_{k}$-move is a generalization of $C_{k}$-move defined by K. Habiro. Habiro showed that two oriented knots are the same up to $C_{k}$-move and ambient isotopy if and only if any Vassiliev invariant of order $\leq k-1$ takes the same value on them. The 'if' part does not hold for two-component links. Our result gives a sufficient condition for spatial graphs to be $C_{k}$-equivalent.
\end{abstract}

\section{Introduction.}

K. Habiro defined a local move, $C_{k}$-move, for each natural number $k$ [2]. It is known that if two embeddings $f$ and $g$ of a graph into the three sphere are the same up to $C_{k}$-move and ambient isotopy, then $g$ can be deformed into a band sum of $f$ with certain $(k+1)$-component links and that changing position of a band and an arc, which is called a band trivialization of $C_{k^{-}}$ move, is realized by $C_{k+1}$-moves and ambient isotopy [17]. This is one of the most important properties of $C_{k}$-move. We consider local moves which have this property. We define $A_{1}$-move as the crossing change and $A_{k+1}$-move as a band trivialization of $A_{k}$-move; see Section 1 for the precise definition. So $A_{k}$-move is a generalization of $C_{k}$-move. In fact, the results for $A_{k}$-move in this paper hold for $C_{k}$-move.

Let $A_{k}$-equivalence denote an equivalence relation given by $A_{k}$-moves and ambient isotopy. Habiro showed that two oriented knots are $C_{k}$-equivalent if and only if they have the same Vassiliev invariants of order $\leq k-1$ [3], [4]. The 'only if' part of this result is true for $A_{k}$-move and for the embeddings of a graph, in particular for links (Theorem 5.1). However the 'if' part does 
not hold for two-component links. For example, the Whitehead link is not $C_{3}$-equivalent to a trivial link because they have different Arf invariants, see [16]. On the other hand, H. Murakami showed in [7] that the Vassiliev invariants of links of order $\leq 2$ are determined by the linking numbers and the second coefficient of the Conway polynomial of each component. Hence, the values of any Vassiliev invariant of order $\leq 2$ of these two links are the same. So we note that Vassiliev invariants of order $\leq k-1$ are not enough to characterize $C_{k}$-equivalent embeddings of a graph.

We will define in Section 1 a finite type invariant of order $(n ; k)$ as a generalization of a Vassiliev invariant and see that if any finite type invariants of order $(1 ; k)$ takes the same value on two $A_{k-1}$-equivalent embeddings of a graph, then they are $A_{k}$-equivalent (Theorem 1.1). While a Vassiliev invariant is defined by the change in its value at every 'wall' corresponding to a crossing change, a finite type invariant of order $(n ; k)$ is defined similarly by 'walls' corresponding to $A_{k}$-moves. A finite type invariant of order $(n ; 1)$ is a Vassiliev invariant of order $\leq n$.

It is shown that the set of $C_{k}$-equivalence classes of knots forms an abelian group under the connected sum [3], [4]. This is also true for $A_{k}$-equivalence classes. Since the connected sum is peculiar to knots, we cannot apply it to embeddings of a graph. In Section 2, we will define a certain geometric sum for the elements in an $A_{k-1}$-equivalence class of the embedding of a graph. Then we will see that the quotient set of the $A_{k-1}$-equivalence class under $A_{k}$-equivalence forms an abelian group (Theorem 2.4).

It is not essential that $A_{1}$-move is the crossing change. This is a big difference between $A_{k}$-move and $C_{k}$-move. We will study a generalization of $A_{k}$-move in Section 4 . For example, if we put $A_{1}$-move to be the \#move defined by Murakami [6], then we get several results similar to that for original $A_{k}$-move.

\section{1. $A_{k}$-moves and finite type invariants.}

Let $B^{3}$ be the oriented unit 3-ball. A tangle is a disjoint union of properly embedded arcs in $B^{3}$. A tangle is trivial if it is contained in a properly embedded 2-disk in $B^{3}$. A trivialization of a tangle $T=t_{1} \cup t_{2} \cup \cdots \cup t_{k}$ is a choice of mutually disjoint disks $D_{1}, D_{2}, \ldots, D_{k}$ in $B^{3}$ such that $D_{i}=\left(D_{i} \cap\right.$ $\left.\partial B^{3}\right) \cup t_{i}$ for $i=1,2, \ldots, k$. It can be shown that in general a trivialization is not unique up to ambient isotopy of $B^{3}$ fixed on the tangle.

Let $T$ and $S$ be tangles, and let $t_{1}, t_{2}, \ldots, t_{k}$ and $s_{1}, s_{2}, \ldots, s_{k}$ be the components of $T$ and $S$ respectively. Suppose that for each $t_{i}$ there exists some $s_{j}$ such that $\partial t_{i}=\partial s_{j}$. Then we call the ordered pair $(T, S)$ a local move, which can be interpreted as substituting $S$ for $T$. Two local moves $(T, S)$ and $\left(T^{\prime}, S^{\prime}\right)$ are equivalent if there exists an orientation preserving homeomorphism $h: B^{3} \longrightarrow B^{3}$ such that $h(T)=T^{\prime}$ and $h(S)$ is ambient isotopic to $S^{\prime}$ relative to $\partial B^{3}$. We consider local moves up to this equivalence. 
Let $(T, S)$ be a local move such that $T$ and $S$ are trivial tangles. First choose a trivialization $D_{1}, D_{2}, \ldots, D_{k}$ of $T$. Each $D_{i}$ intersects $\partial B^{3}$ in an $\operatorname{arc} \gamma_{i}$. Let $E_{i}$ be a small regular neighbourhood of $\gamma_{i}$ in $\partial B^{3}$. We devide the circle $\partial E_{i}$ into two arcs $\alpha_{i}$ and $\beta_{i}$ such that $\alpha_{i} \cap \beta_{i}=\partial \alpha_{i}=\partial \beta_{i}$. By slightly perturbing int $\alpha_{i}$ and int $\beta_{i}$ into the interior of $B^{3}$ on either side of $D_{i}$, we obtain properly embedded $\operatorname{arcs} \widetilde{\alpha}_{i}$ and $\widetilde{\beta}_{i}$. We consider $k$ local moves $\left(S \cup \widetilde{\alpha}_{i}, S \cup \widetilde{\beta}_{i}\right)(i=1,2, \ldots, k)$ and call them the band trivializations of the local move $(T, S)$ with respect to the trivialization $D_{1}, D_{2}, \ldots, D_{k}$. Note that both $S \cup \widetilde{\alpha}_{i}$ and $S \cup \widetilde{\beta}_{i}$ are trivial tangles.

We now inductively define a sequence of local moves on trivial tangles in $B^{3}$ which depend on the choice of trivialization. An $A_{1}$-move is the crossing change shown in Figure 1.1. Suppose that $A_{k}$-moves are defined and there are $l A_{k}$-moves $\left(T_{1}, S_{1}\right),\left(T_{2}, S_{2}\right), \ldots,\left(T_{l}, S_{l}\right)$ up to equivalence. For each $A_{k}$-move $\left(T_{i}, S_{i}\right)(i=1,2, \ldots, l)$, we choose a single trivialization $\tau_{i}=\left\{D_{i, 1}, D_{i, 2}, \ldots, D_{i, k+1}\right\}$ of $T_{i}$ and fix it. (The choice of $\tau_{i}$ is independent of the trivialization that is chosen to define $A_{k}$-move $\left(T_{i}, S_{i}\right)$.) Then the band trivializations of $\left(T_{i}, S_{i}\right)$ with respect to the trivialization $\tau_{i}$ are called $A_{k+1}\left(\tau_{i}\right)$-moves and these $A_{k+1}\left(\tau_{i}\right)$-moves $(i=1,2, \ldots, l)$ are called $A_{k+1}\left(\tau_{1}, \tau_{2}, \ldots, \tau_{l}\right)$-moves. Note that the number of $A_{k+1}\left(\tau_{1}, \tau_{2}, \ldots, \tau_{l}\right)$ moves is at most $l(k+1)$ up to equivalence. Although the choice of trivializations is important for the definition of $A_{k}$-move, our proof is the same for every choice. Therefore the results of this paper hold for every choice of trivializations $\tau_{1}, \tau_{2}, \ldots, \tau_{l}$. So we denote $A_{k+1}\left(\tau_{1}, \tau_{2}, \ldots, \tau_{l}\right)$-move simply as $A_{k+1}$-move. It is known that $C_{k}$-move defined by Habiro is a special case of $A_{k}$-move for certain choices of trivializations; see [2], [10]. We will see that $A_{k}$-move, as well as $C_{k}$-move, has the property mentioned in Introduction (Proposition 2.1 and Lemma 2.2).

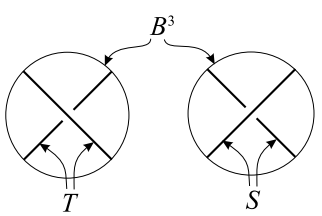

Figure 1.1.

Examples. (1) The trivialization of a tangle in Figure 1.1 is unique up to ambient isotopy. Therefore we have any band trivialization of an $A_{1}$-move is equivalent to the local move in Figure 1.2-(i). Thus $A_{2}$-move is unique up to equivalence. It is not hard to see that an $A_{2}$-move is equivalent to the delta move in Figure 1.2-(ii) defined by H. Murakami and Y. Nakanishi [8], and then it is equivalent to the local move in Figure 1.2-(iii). 
(2) If we choose a trivialization for the $A_{2}$-move as in Figure 1.3-(i), then, by the symmetry of the $A_{2}$-move, any $A_{3}$-move is equivalent to the local move in Figure 1.3-(ii).

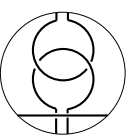

(i)

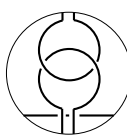

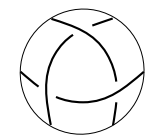

(ii)

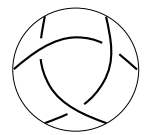

ii)

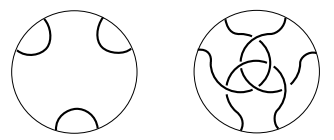

(iii)

Figure 1.2.

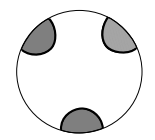

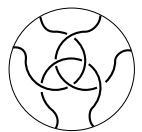

(i)

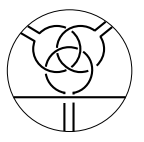

(ii)

Figure 1.3.

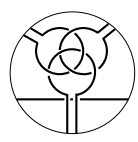

Figure 1.3.

A local move $(S, T)$ is called the inverse of a local move $(T, S)$. It is clear that the inverse of an $A_{1}$-move is again an $A_{1}$-move. By the definition of $A_{k}$-move, we see that the inverse of an $A_{k}$-move with $k \geq 2$ is equivalent to itself.

Let $(T, S)$ be an $A_{k}$-move and $D_{1}, D_{2}, \ldots, D_{k+1}$ the fixed trivialization of $T=t_{1} \cup t_{2} \cup \cdots \cup t_{k+1}$. We set $\alpha=\partial B^{3} \cap\left(D_{1} \cup D_{2} \cup \cdots \cup D_{k+1}\right)$ and $\beta=S$. A link $L$ in $S^{3}$ is called type $k$ if there is an orientation preserving embedding $\varphi: B^{3} \longrightarrow S^{3}$ such that $L=\varphi(\alpha \cup \beta)$. Then the pair $(\alpha, \beta)$ is called a link model of $L$.

We now define an equivalence relation on spatial graphs by $A_{k}$-move. Let $G$ be a finite graph. Let $V(G)$ denote the set of the vertices of $G$. Let $f, g: G \longrightarrow S^{3}$ be embeddings. We say that $f$ and $g$ are related by an $A_{k^{-}}$ move if there is an $A_{k}$-move $(T, S)$ and an orientation preserving embedding $\varphi: B^{3} \longrightarrow S^{3}$ such that:

(i) If $f(x) \neq g(x)$ then both $f(x)$ and $g(x)$ are contained in $\varphi\left(\operatorname{int} B^{3}\right)$,

(ii) $f(V(G))=g(V(G))$ is disjoint from $\varphi\left(B^{3}\right)$, and

(iii) $f(G) \cap \varphi\left(B^{3}\right)=\varphi(T)$ and $g(G) \cap \varphi\left(B^{3}\right)=\varphi(S)$.

We also say that $g$ is obtained from $f$ by an application of $(T, S)$. We define $A_{k}$-equivalence as an equivalence relation on the set of all embeddings of $G$ into $S^{3}$ given by the relation above and ambient isotopy. For an embedding $f: G \longrightarrow S^{3}$, let $[f]_{k}$ denote the $A_{k}$-equivalence class of $f$. By the definition of $A_{k}$-move we see that an application of an $A_{k+1}$-move is realized by two applications of $A_{k}$-move and ambient isotopy. Thus $A_{k+1}$-equivalence implies $A_{k}$-equivalence. In other words we have $[f]_{1} \supset[f]_{2} \supset \cdots \supset[f]_{k} \supset$ $[f]_{k+1} \supset \cdots$. 
Let $f: G \longrightarrow S^{3}$ be an embedding, $L_{i}$ links of type $k$ and $\left(\alpha_{i}, \beta_{i}\right)$ their link models $(i=1,2, \ldots, n)$. Let $I=[0,1]$ be the unit closed interval. An embedding $g: G \longrightarrow S^{3}$ is called a band sum of $f$ with $L_{1}, L_{2}, \ldots, L_{n}$ if there are mutually disjoint embeddings $b_{i j}: I \times I \longrightarrow S^{3}(i=1,2, \ldots, n, j=$ $1,2, \ldots, k+1)$ and mutually disjoint orientation preserving embeddings $\varphi_{i}$ : $B^{3} \longrightarrow S^{3}-f(G)$ with $L_{i}=\varphi_{i}\left(\alpha_{i} \cup \beta_{i}\right)(i=1,2, \ldots, n)$ such that the following (i) and (ii) hold:

(i) $b_{i j}(I \times I) \cap f(G)=b_{i j}(I \times I) \cap f(G-V(G))=b_{i j}(I \times\{0\})$ and $b_{i j}(I \times I) \cap\left(\bigcup_{l} \varphi_{l}\left(B^{3}\right)\right)=b_{i j}(I \times\{1\})$ is a component of $\varphi_{i}\left(\alpha_{i}\right)$ for any $i, j(i=1,2, \ldots, n, j=1,2, \ldots, k+1)$.

(ii) $f(x)=g(x)$ if $f(x)$ is not contained in $\bigcup_{i, j} b_{i j}(I \times\{0\})$ and

$$
g(G)=\left(f(G) \cup \bigcup_{i} L_{i}-\bigcup_{i, j} b_{i j}(I \times \partial I)\right) \cup \bigcup_{i, j} b_{i j}(\partial I \times I) .
$$

Then we denote $g$ by $F\left(f ;\left\{L_{1}, L_{2}, \ldots, L_{n}\right\},\left\{B_{1}, B_{2}, \ldots, B_{n}\right\}\right)$, where $B_{i}=$ $b_{i 1}(I \times I) \cup b_{i 2}(I \times I) \cup \cdots \cup b_{i k+1}(I \times I)(i=1,2, \ldots, n)$. We call each $b_{i j}(I \times I)$ a band. We call each $\varphi_{i}\left(B^{3}\right)$ an associated ball of $L_{i}$. See Figure 1.4 for an example of a band sum of an embedding $f$ with links $L_{1}, L_{2}, L_{3}$ of type 3 .
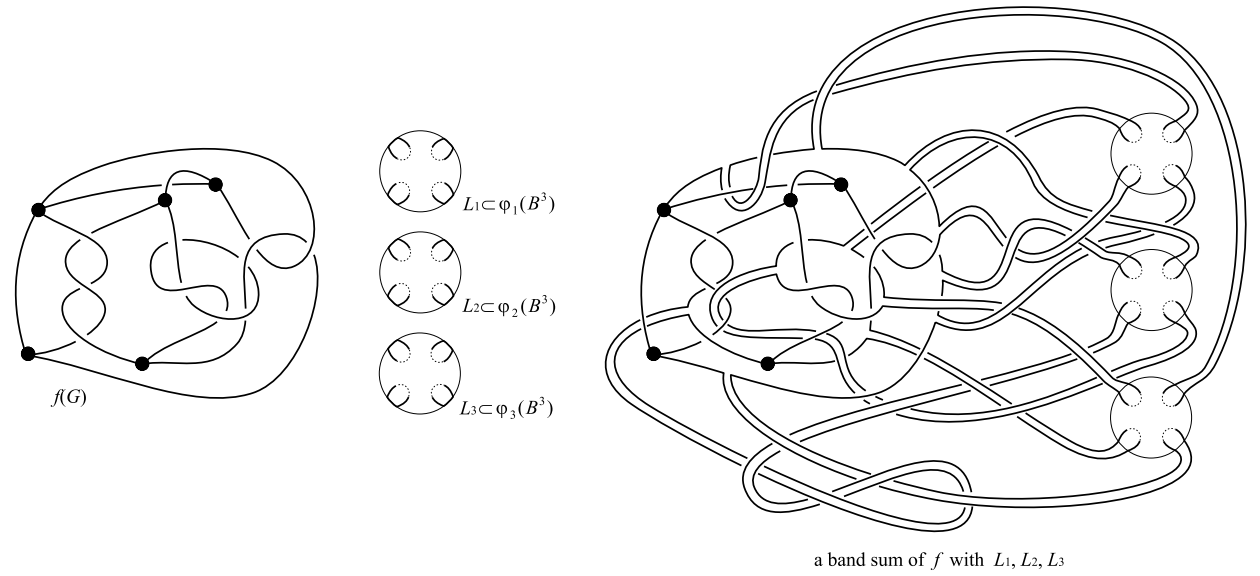

Figure 1.4.

Remark. It follows from the definition that if $g$ is a band sum of $f$ with some links of type $k$, then $g$ is $A_{k}$-equivalent to $f$. The converse is also true and will be shown in Proposition 2.1. In Lemma 2.2, we show that the position of a band is changeable up to $A_{k+1}$-equivalence. The origin of the name 'band trivialization' comes from this fact.

Let $h: G \longrightarrow S^{3}$ be an embedding and $H$ an abelian group. Let $\varphi:[h]_{k-1} \longrightarrow H$ be an invariant. We say that $\varphi$ is a finite type invariant of order $(n ; k)$ if for any embedding $f \in[h]_{k-1}$ and any band 
sum $F\left(f ;\left\{L_{1}, L_{2}, \ldots, L_{n+1}\right\},\left\{B_{1}, B_{2}, \ldots, B_{n+1}\right\}\right)$ of $f$ with links $L_{1}, L_{2}, \ldots$, $L_{n+1}$ of type $k-1$,

$$
\sum_{X \subset\{1,2, \ldots, n+1\}}(-1)^{|X|} \varphi\left(F\left(f ; \bigcup_{i \in X}\left\{L_{i}\right\}, \bigcup_{i \in X}\left\{B_{i}\right\}\right)\right)=0 \in H,
$$

where the sum is taken over all subsets, including the empty set, and $|X|$ is the number of the elements in $X$.

In the next section we show the following theorem:

Theorem 1.1. Let $f, g: G \longrightarrow S^{3}$ be $A_{k-1}$-equivalent embeddings. Then they are $A_{k}$-equivalent if and only if $\varphi(f)=\varphi(g)$ for any finite type $A_{k}$ equivalence invariant $\varphi$ of order $(1 ; k)$.

Note that finite type invariants of order $(n ; 2)$ coincide with Vassiliev invariants of order $n$. It is shown in [5, Theorem 1.1, Theorem 1.3] that two embeddings of a finite graph $G$ into $S^{3}$ are $A_{2}$-equivalent if and only if they have the same $\mathrm{Wu}$ invariant [18]. It follows from $[\mathbf{1 4}$, Section 2] that $\mathrm{Wu}$ invariant is a finite type invariant of order $(1 ; 2)$. Since two embeddings are always $A_{1}$-equivalent, we have the following corollary:

Corollary 1.2. Let $f, g: G \longrightarrow S^{3}$ be embeddings. Then the following conditions are mutually equivalent:

(i) $f$ and $g$ are $A_{2}$-equivalent.

(ii) $f$ and $g$ have the same $W u$ invariant.

(iii) $\varphi(f)=\varphi(g)$ for any Vassiliev invariant $\varphi$ of order 1 .

In Section 5 we show the following proposition:

Proposition 1.3. Let $\varphi$ be a Vassiliev invariant of order $(n+1)(k-1)-1$. Then $\varphi$ is a finite type invariant of order $(n ; k)$.

\section{2. $A_{k}$-equivalence group of spatial graphs.}

The following proposition is a natural generalization of [21, Lemma] and stems from the fact that a knot with the unknotting number $u$ can be unknotted by changing $u$ crossings of a regular diagram of it [12] and [19].

Proposition 2.1. Let $f, g: G \longrightarrow S^{3}$ be embeddings. If $f$ and $g$ are $A_{k^{-}}$ equivalent, then $g$ is ambient isotopic to a band sum of $f$ with some links of type $k$.

Proof. We consider the embeddings up to ambient isotopy for simplicity. By the assumption there is a finite sequence of embeddings $f=f_{0}, f_{1}, \ldots, f_{n}=$ $g$ and orientation preserving embeddings $\varphi_{1}, \varphi_{2}, \ldots, \varphi_{n}: B^{3} \longrightarrow S^{3}$ such that $\left(\varphi_{i}^{-1}\left(f_{i-1}(G)\right), \varphi_{i}^{-1}\left(f_{i}(G)\right)\right)$ is an $A_{k}$-move for each $i$. We shall prove this proposition by induction on $n$. 


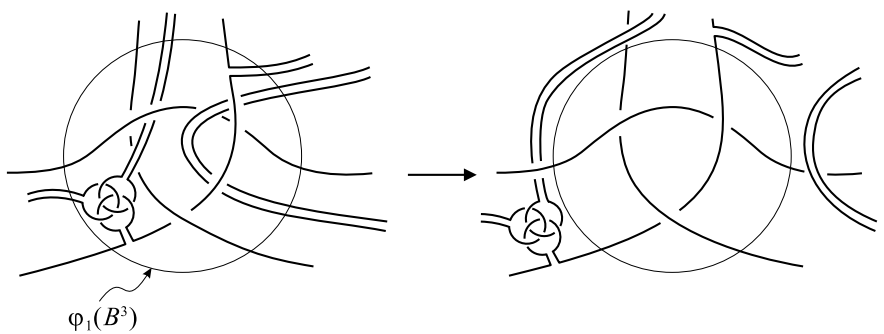

Figure 2.1.

First we consider the case $n=1$. Let $D_{1}, D_{2}, \ldots, D_{k+1}$ be the fixed trivialization of the tangle $\varphi_{1}^{-1}\left(f_{0}(G)\right)$ and $\gamma_{j}=D_{j} \cap \partial B^{3}(j=1,2, \ldots, k+$ 1). Then $L=\bigcup_{j} \varphi_{1}\left(\gamma_{j}\right) \cup\left(\varphi_{1}\left(B^{3}\right) \cap f_{1}(G)\right)$ is a link of type $k$. By taking a small one-sided collar for each $\varphi_{1}\left(\gamma_{j}\right)$ in $S^{3}-\varphi_{1}\left(\operatorname{int} B^{3}\right)$, we have mutually disjoint embeddings $b_{j}: I \times I \longrightarrow S^{3}(j=1,2, \ldots, k+1)$ such that $b_{j}(I \times$ $I) \cap \varphi_{1}\left(B^{3}\right)=b_{j}(I \times\{1\})=\varphi_{1}\left(\gamma_{j}\right)$ and $b_{j}(I \times I) \cap f_{0}(G)=b_{j}(I \times I) \cap$ $f_{1}(G)=b_{j}(\partial I \times I)$. Then we deform $f_{0}$ up to ambient isotopy along the disk $b_{j}(I \times I) \cup \varphi_{1}\left(D_{j}\right)$ such that $b_{j}(I \times I) \cap f_{0}(G)=b_{j}(I \times\{0\})$ for each $j$. Then we have a required band sum $g=F\left(f_{0} ;\{L\},\{B\}\right)$, where $B=$ $b_{1}(I \times I) \cup b_{2}(I \times I) \cup \cdots \cup b_{k+1}(I \times I)$.

Next suppose that $n>1$. By the hypothesis of our induction, $g$ is a band sum $F\left(f_{1} ; \mathcal{L}, \mathcal{B}\right)$, where $\mathcal{L}=\left\{L_{1}, L_{2}, \ldots, L_{n-1}\right\}$ is a set of links of type $k$, $\mathcal{B}=\left\{B_{1}, B_{2}, \ldots, B_{n-1}\right\}$ and each $B_{i}$ is a union of bands attaching to $L_{i}$. Deform $F\left(f_{1} ; \mathcal{L}, \mathcal{B}\right)$ up to ambient isotopy keeping the image $f_{1}(G)$ so that neither the associated balls of $\mathcal{L}$ nor the bands in $\mathcal{B}$ intersect $\varphi_{1}\left(B^{3}\right)$. Note that this deformation is possible, since the tangle $\varphi_{1}^{-1}\left(f_{1}(G)\right)$ is trivial. In fact, sweeping out the associated balls, band-slidings and sweeping out the bands are sufficient. See Figure 2.1. Then by the same arguments as that in the case $n=1$, we find that $f_{1}$ is a band sum $F(f ;\{L\},\{B\})$. Then we have

$$
F(F(f ;\{L\},\{B\}) ; \mathcal{L}, \mathcal{B})=F(f ;\{L\} \cup \mathcal{L},\{B\} \cup \mathcal{B}) .
$$

This completes the proof.

As we mentioned before, the origin of the name 'band trivialization' comes from the following lemma:

Lemma 2.2. The moves in Figures 2.2-(i), (ii), (iii) and (iv) are realized by $A_{k+1}$-moves.

Proof. The move in Figure 2.2-(i) is just a band trivialization of an $A_{k}$-move. Hence by the definition it is an $A_{k+1}$-move. It is easy to see that the moves in Figures 2.2-(ii) and (iii) are generated by the moves in Figure 2.2-(i). To see that the move in Figure 2.2-(iv) is realized by $A_{k+1}$-moves, we first 


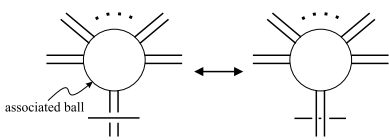

(i)

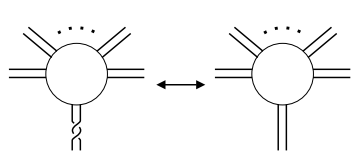

(iii)

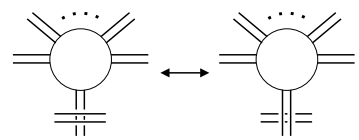

(ii)

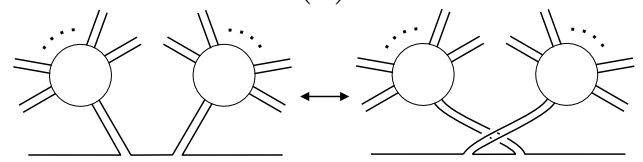

(iv)

\section{Figure 2.2.}

slide the bands as illustrated in Figure 2.3, and then perform the moves in Figure 2.2-(i).
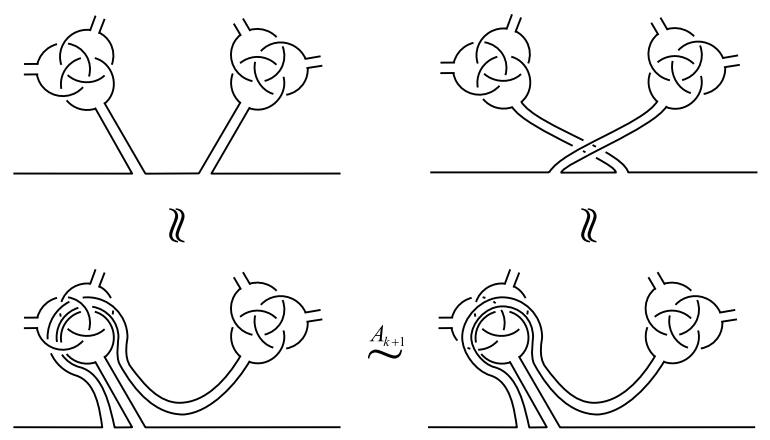

Figure 2.3.

Let $h: G \longrightarrow S^{3}$ be an embedding and let $\left[f_{1}\right]_{k},\left[f_{2}\right]_{k} \in[h]_{k-1} /\left(A_{k}\right.$-equivalence), where $[h]_{k-1} /\left(A_{k}\right.$-equivalence $)$ denotes the set of $A_{k}$-equivalence classes in $[h]_{k-1}$. Since both $f_{1}$ and $f_{2}$ are $A_{k-1}$-equivalent to $h$, by Proposition 2.1 , there are band sums $F\left(h ; \mathcal{L}_{i}, \mathcal{B}_{i}\right) \in\left[f_{i}\right]_{k}$ of $h$ with links $\mathcal{L}_{i}$ of type $k-1(i=1,2)$. Suppose that the bands in $\mathcal{B}_{1}$ and the associated balls of $\mathcal{L}_{1}$ are disjoint from the bands in $\mathcal{B}_{2}$ and the associated balls of $\mathcal{L}_{2}$. Note that up to slight ambient isotopy of $F\left(h ; \mathcal{L}_{2}, \mathcal{B}_{2}\right)$ that preserves $h(G)$ we can always choose the bands and the associated balls so that they satisfy this condition. In the following we assume this condition without explicit mention. Then we have a new band sum $F\left(h ; \mathcal{L}_{1} \cup \mathcal{L}_{2}, \mathcal{B}_{1} \cup \mathcal{B}_{2}\right)$. We define

$$
\left[f_{1}\right]_{k}+{ }_{h}\left[f_{2}\right]_{k}=\left[F\left(h ; \mathcal{L}_{1} \cup \mathcal{L}_{2}, \mathcal{B}_{1} \cup \mathcal{B}_{2}\right)\right]_{k} .
$$

Lemma 2.3. The sum ' $+h$ ' above is well-defined.

Proof. It is sufficient to show for two embeddings $F\left(h ; \mathcal{L}_{1}, \mathcal{B}_{1}\right), F\left(h ; \mathcal{L}_{1}^{\prime}, \mathcal{B}_{1}^{\prime}\right) \in$ $\left[f_{1}\right]_{k}$ that $F\left(h ; \mathcal{L}_{1} \cup \mathcal{L}_{2}, \mathcal{B}_{1} \cup \mathcal{B}_{2}\right)$ and $F\left(h ; \mathcal{L}_{1}^{\prime} \cup \mathcal{L}_{2}, \mathcal{B}_{1}^{\prime} \cup \mathcal{B}_{2}\right)$ are $A_{k^{-}}$equivalent. 
Consider a sequence of ambient isotopies and applications of $A_{k}$-moves that deforms $F\left(h ; \mathcal{L}_{1}, \mathcal{B}_{1}\right)$ into $F\left(h ; \mathcal{L}_{1}^{\prime}, \mathcal{B}_{1}^{\prime}\right)$. We consider this sequence of deformations together with the links in $\mathcal{L}_{2}$ and the bands in $\mathcal{B}_{2}$. Whenever we apply an $A_{k}$-move we deform the associated balls of $\mathcal{L}_{2}$ and the bands in $\mathcal{B}_{2}$ up to ambient isotopy so that they are away from the 3-ball within which the $A_{k}$-move is applied. Thus $F\left(h ; \mathcal{L}_{1} \cup \mathcal{L}_{2}, \mathcal{B}_{1} \cup \mathcal{B}_{2}\right)=F\left(F\left(h ; \mathcal{L}_{1}, \mathcal{B}_{1}\right) ; \mathcal{L}_{2}, \mathcal{B}_{2}\right)$ is $A_{k}$-equivalent to a band sum $F\left(F\left(h ; \mathcal{L}_{1}^{\prime}, \mathcal{B}_{1}^{\prime}\right) ; \mathcal{L}_{2}^{\prime}, \mathcal{B}_{2}^{\prime}\right)$ for some $\mathcal{L}_{2}^{\prime}$ and $\mathcal{B}_{2}^{\prime}$. Compare the band sums $F\left(F\left(h ; \mathcal{L}_{1}^{\prime}, \mathcal{B}_{1}^{\prime}\right) ; \mathcal{L}_{2}^{\prime}, \mathcal{B}_{2}^{\prime}\right)$ and $F\left(h ; \mathcal{L}_{1}^{\prime} \cup \mathcal{L}_{2}, \mathcal{B}_{1}^{\prime} \cup \mathcal{B}_{2}\right)=$ $F\left(F\left(h ; \mathcal{L}_{1}^{\prime}, \mathcal{B}_{1}^{\prime}\right) ; \mathcal{L}_{2}, \mathcal{B}_{2}\right)$. We have that the links in $\mathcal{L}_{2}^{\prime}$ are ambient isotopic to the links in $\mathcal{L}_{2}$. It follows from Lemma 2.2 that the bands in $\mathcal{B}_{2}^{\prime}$ can be deformed into the position of the bands in $\mathcal{B}_{2}$ by band slidings and $A_{k}$-moves. Thus these two are $A_{k}$-equivalent.

Theorem 2.4. The set $[h]_{k-1} /\left(A_{k}\right.$-equivalence $)$ forms an abelian group under ' ${ }_{h}$ ' with the unit element $[h]_{k}$.

We denote this group by $\mathcal{G}_{k}(h ; G)$ and call it the $A_{k}$-equivalence group of the spatial embeddings of $G$ with the unit element $[h]_{k}$.

Remark. Note that for any graph $G$ and any embedding $h: G \longrightarrow S^{3}$, $[h]_{1}$ is equal to the set of all embeddings of $G$ into $S^{3}$. In [20], the second author called $\mathcal{G}_{2}(h ; G)$ a graph homology group and gave a practical method of calculating this group.

Proof. We consider embeddings up to ambient isotopy for simplicity. It is sufficient to show that for any $[f]_{k} \in[h]_{k-1} /\left(A_{k}\right.$-equivalence), there is an inverse of $[f]_{k}$. Since $f$ and $h$ are $A_{k-1}$-equivalent, by Proposition 2.1, $f$ and $h$ are band sums $F(h ; \mathcal{L}, \mathcal{B})$ and $F\left(f ; \mathcal{L}^{\prime}, \mathcal{B}^{\prime}\right)$ respectively, where $\mathcal{L}$ and $\mathcal{L}^{\prime}$ are sets of links of type $k-1$. Thus we have $h=F\left(F(h ; \mathcal{L}, \mathcal{B}) ; \mathcal{L}^{\prime}, \mathcal{B}^{\prime}\right)$. Then, by using Lemma 2.2, we deform the associated balls of $\mathcal{L}^{\prime}$ and the bands in $\mathcal{B}^{\prime}$ up to $A_{k}$-equivalence so that they are disjoint form the associated balls of $\mathcal{L}$ and the bands in $\mathcal{B}$. Thus we see that $h=F\left(F(h ; \mathcal{L}, \mathcal{B}) ; \mathcal{L}^{\prime}, \mathcal{B}^{\prime}\right)$ is $A_{k}$-equivalent to a band sum $F\left(h ; \mathcal{L} \cup \mathcal{L}^{\prime \prime}, \mathcal{B} \cup \mathcal{B}^{\prime \prime}\right)$ for some $\mathcal{L}^{\prime \prime}$ and $\mathcal{B}^{\prime \prime}$ (for example see Figure 2.4). Thus we have

$$
\begin{aligned}
{[f]_{k}+{ }_{h}\left[F\left(h ; \mathcal{L}^{\prime \prime}, \mathcal{B}^{\prime \prime}\right)\right]_{k} } & =[F(h ; \mathcal{L}, \mathcal{B})]_{k}+{ }_{h}\left[F\left(h ; \mathcal{L}^{\prime \prime}, \mathcal{B}^{\prime \prime}\right)\right]_{k} \\
& =\left[F\left(h ; \mathcal{L} \cup \mathcal{L}^{\prime \prime}, \mathcal{B} \cup \mathcal{B}^{\prime \prime}\right)\right]_{k} \\
& =[h]_{k} .
\end{aligned}
$$

This implies that $\left[F\left(h ; \mathcal{L}^{\prime \prime}, \mathcal{B}^{\prime \prime}\right)\right]_{k}$ is an inverse of $[f]_{k}$.

Theorem 2.5. Let $h_{1}, h_{2}: G \longrightarrow S^{3}$ be $A_{k-1}$-equivalent embeddings. Then the groups $\mathcal{G}_{k}\left(h_{1} ; G\right)$ and $\mathcal{G}_{k}\left(h_{2} ; G\right)$ are isomorphic.

Proof. We define a map $\phi: \mathcal{G}_{k}\left(h_{1} ; G\right) \longrightarrow \mathcal{G}_{k}\left(h_{2} ; G\right)$ by $\phi\left([f]_{k}\right)=[f]_{k}-{ }_{h_{2}}$ $\left[h_{1}\right]_{k}$, where $[x]_{k}-{ }_{h_{2}}[y]_{k}$ denotes $[x]_{k}+{ }_{h_{2}}\left(-[y]_{k}\right)$. Clearly this map is a bijection. We shall prove that $\phi$ is a homomorphism. Let $\left[f_{i}\right]_{k} \in \mathcal{G}_{k}\left(h_{1} ; G\right)(i=$ 

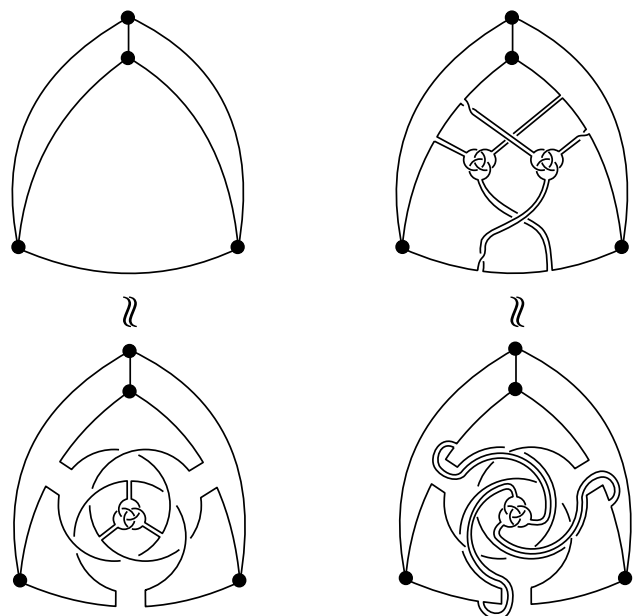

१

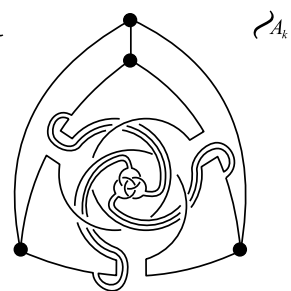

Figure 2.4.

$1,2)$. Then $f_{i}=F\left(h_{1} ; \mathcal{L}_{i}, \mathcal{B}_{i}\right)$ where $\mathcal{L}_{i}$ is a set of links of type $k-1(i=1,2)$. Since $h_{1}$ and $h_{2}$ are $A_{k-1}$-equivalent we see that $h_{1}=F\left(h_{2} ; \mathcal{L}, \mathcal{B}\right)$ where $\mathcal{L}$ is a set of links of type $k-1$. Thus we have $f_{i}=F\left(F\left(h_{2} ; \mathcal{L}, \mathcal{B}\right) ; \mathcal{L}_{i}, \mathcal{B}_{i}\right)(i=$ $1,2)$. By using Lemma 2.2 , we deform $f_{i}$ up to $A_{k}$-equivalence so that the associated balls of $\mathcal{L}_{i}$ and the bands in $\mathcal{B}_{i}$ are disjoint from the associated balls of $\mathcal{L}$ and the bands in $\mathcal{B}$ for $i=1,2$. We may further assume that the associated balls of $\mathcal{L}_{1}$ and the bands in $\mathcal{B}_{1}$ are disjoint from the associated balls of $\mathcal{L}_{2}$ and the bands in $\mathcal{B}_{2}$. Then we have

$$
\begin{aligned}
\phi\left(\left[f_{1}\right]_{k}+{ }_{h_{1}}\left[f_{2}\right]_{k}\right) & =\phi\left(\left[F\left(F\left(h_{2} ; \mathcal{L}, \mathcal{B}\right) ; \mathcal{L}_{1} \cup \mathcal{L}_{2}, \mathcal{B}_{1} \cup \mathcal{B}_{2}\right)\right]_{k}\right) \\
& =\left[F\left(F\left(h_{2} ; \mathcal{L}, \mathcal{B}\right) ; \mathcal{L}_{1} \cup \mathcal{L}_{2}, \mathcal{B}_{1} \cup \mathcal{B}_{2}\right)\right]_{k}-{ }_{h_{2}}\left[h_{1}\right]_{k} \\
& =\left[F\left(h_{2} ; \mathcal{L} \cup \mathcal{L}_{1} \cup \mathcal{L}_{2}, \mathcal{B} \cup \mathcal{B}_{1} \cup \mathcal{B}_{2}\right)\right]_{k}-{ }_{h_{2}}\left[F\left(h_{2} ; \mathcal{L}, \mathcal{B}\right)\right]_{k} \\
& =\left[F\left(h_{2} ; \mathcal{L}_{1} \cup \mathcal{L}_{2}, \mathcal{B}_{1} \cup \mathcal{B}_{2}\right)\right]_{k},
\end{aligned}
$$

and for each $i(i=1,2)$,

$$
\begin{aligned}
\phi\left(\left[f_{i}\right]_{k}\right) & =\phi\left(\left[F\left(F\left(h_{2} ; \mathcal{L}, \mathcal{B}\right) ; \mathcal{L}_{i}, \mathcal{B}_{i}\right)\right]_{k}\right) \\
& =\left[F\left(F\left(h_{2} ; \mathcal{L}, \mathcal{B}\right) ; \mathcal{L}_{i}, \mathcal{B}_{i}\right)\right]_{k}-{ }_{h_{2}}\left[h_{1}\right]_{k} \\
& =\left[F\left(h_{2} ; \mathcal{L} \cup \mathcal{L}_{i}, \mathcal{B} \cup \mathcal{B}_{i}\right)\right]_{k}-{ }_{h_{2}}\left[F\left(h_{2} ; \mathcal{L}, \mathcal{B}\right)\right]_{k} \\
& =\left[F\left(h_{2} ; \mathcal{L}_{i}, \mathcal{B}_{i}\right)\right]_{k} .
\end{aligned}
$$


Thus we have $\phi\left(\left[f_{1}\right]_{k}+{ }_{h_{1}}\left[f_{2}\right]_{k}\right)=\phi\left(\left[f_{1}\right]_{k}\right)+{ }_{h_{2}} \phi\left(\left[f_{2}\right]_{k}\right)$.

Proposition 2.6. The projection $p:[h]_{k-1} \longrightarrow[h]_{k-1} /\left(A_{k}\right.$-equivalence $)=$ $\mathcal{G}_{k}(h ; G)$ is a finite type $A_{k}$-equivalence invariant of order $(1 ; k)$.

Proof. It is clear that $p$ is an $A_{k}$-equivalence invariant. We shall prove that $p$ is finite type of order $(1 ; k)$. Let $f \in[h]_{k-1}$ be an embedding and $F\left(f ;\left\{L_{1}, L_{2}\right\},\left\{B_{1}, B_{2}\right\}\right)$ a band sum of $f$ with links $L_{1}, L_{2}$ of type $k-1$. Then it is sufficient to show that

$$
\sum_{X \subset\{1,2\}}(-1)^{|X|} p\left(F\left(f ; \bigcup_{i \in X}\left\{L_{i}\right\}, \bigcup_{i \in X}\left\{B_{i}\right\}\right)\right)=[h]_{k} .
$$

Let $\phi: \mathcal{G}_{k}(f ; G) \longrightarrow \mathcal{G}_{k}(h ; G)$ be the isomorphism defined by $\phi\left([g]_{k}\right)=$ $[g]_{k}-{ }_{h}[f]_{k}$. Then we have

$$
\begin{aligned}
\phi( & {[F(f ; \emptyset, \emptyset)]_{k}-{ }_{f}\left[F\left(f ;\left\{L_{1}\right\},\left\{B_{1}\right\}\right)\right]_{k} } \\
& \left.\quad-{ }_{f}\left[F\left(f ;\left\{L_{2}\right\},\left\{B_{2}\right\}\right)\right]_{k}+{ }_{f}\left[F\left(f ;\left\{L_{1}, L_{2}\right\},\left\{B_{1}, B_{2}\right\}\right)\right]_{k}\right) \\
= & \left([F(f ; \emptyset, \emptyset)]_{k}-{ }_{h}[f]_{k}\right)-_{h}\left(\left[F\left(f ;\left\{L_{1}\right\},\left\{B_{1}\right\}\right)\right]_{k}-{ }_{h}[f]_{k}\right) \\
& -{ }_{h}\left(\left[F\left(f ;\left\{L_{2}\right\},\left\{B_{2}\right\}\right)\right]_{k}-{ }_{h}[f]_{k}\right)+_{h}\left(\left[F\left(f ;\left\{L_{1}, L_{2}\right\},\left\{B_{1}, B_{2}\right\}\right)\right]_{k}-{ }_{h}[f]_{k}\right) \\
= & {[F(f ; \emptyset, \emptyset)]_{k}-{ }_{h}\left[F\left(f ;\left\{L_{1}\right\},\left\{B_{1}\right\}\right)\right]_{k}-{ }_{h}\left[F\left(f ;\left\{L_{2}\right\},\left\{B_{2}\right\}\right)\right]_{k} } \\
& +{ }_{h}\left[F\left(f ;\left\{L_{1}, L_{2}\right\},\left\{B_{1}, B_{2}\right\}\right)\right]_{k} \\
= & \sum_{X \subset\{1,2\}}(-1)^{|X|} p\left(F\left(f ; \bigcup_{i \in X}\left\{L_{i}\right\}, \bigcup_{i \in X}\left\{B_{i}\right\}\right)\right) .
\end{aligned}
$$

Since

$$
\left[F\left(f ;\left\{L_{1}, L_{2}\right\},\left\{B_{1}, B_{2}\right\}\right)\right]_{k}=\left[F\left(f ;\left\{L_{1}\right\},\left\{B_{1}\right\}\right)\right]_{k}+_{f}\left[F\left(f ;\left\{L_{2}\right\},\left\{B_{2}\right\}\right)\right]_{k},
$$

we have

$$
\begin{aligned}
\phi & \left([F(f ; \emptyset, \emptyset)]_{k}-{ }_{f}\left[F\left(f ;\left\{L_{1}\right\},\left\{B_{1}\right\}\right)\right]_{k}-{ }_{f}\left[F\left(f ;\left\{L_{2}\right\},\left\{B_{2}\right\}\right)\right]_{k}\right. \\
& \left.\quad{ }_{f}\left[F\left(f ;\left\{L_{1}, L_{2}\right\},\left\{B_{1}, B_{2}\right\}\right)\right]_{k}\right) \\
= & \phi\left([f]_{k}\right) \\
= & {[h]_{k} . }
\end{aligned}
$$

This completes the proof.

Proof of Theorem 1.1. The 'only if' part is clear. We show the 'if' part. Let $f$ and $g$ be embeddings in $[h]_{k-1}$. Suppose that any finite type invariant of order $(1 ; k)$ takes the same value on $f$ and $g$. Then by Proposition 2.6 we have $p(f)=p(g)$, where $p:[h]_{k-1} \longrightarrow[h]_{k-1} /\left(A_{k}\right.$-equivalence $)=\mathcal{G}_{k}(h ; G)$ is the projection. Hence we have $[f]_{k}=[g]_{k}$. This completes the proof. 


\section{3. $A_{k}$-equivalence group of knots.}

In this section we only consider the case that the graph $G$ is homeomorphic to a disjoint union of circles. Let $G=S_{1}^{1} \cup S_{2}^{1} \cup \cdots \cup S_{\mu}^{1}$. Then there is a natural correspondence between the ambient isotopy classes of the embeddings of $G$ into $S^{3}$ and the ambient isotopy classes of the ordered oriented $\mu$-component links in $S^{3}$. Therefore instead of specifying an embedding $h: S_{1}^{1} \cup S_{2}^{1} \cup \cdots \cup$ $S_{\mu}^{1} \longrightarrow S^{3}$, we denote by $L$ the image $h\left(S_{1}^{1} \cup S_{2}^{1} \cup \cdots \cup S_{\mu}^{1}\right)$ and consider it together with the orientation of each component and the ordering of the components. Thus $\mathcal{G}_{k}(L)$ denotes the $A_{k}$-equivalence group $\mathcal{G}_{k}\left(h ; S_{1}^{1} \cup S_{2}^{1} \cup\right.$ $\left.\cdots \cup S_{\mu}^{1}\right)$ with the unit element $[h]_{k}$.

Theorem 3.1. Let $O$ be a trivial knot. Then for any oriented knot $K$, $\mathcal{G}_{k}(O)$ and $\mathcal{G}_{k}(K)$ are isomorphic.

Remark. For a graph $G\left(\neq S^{1}\right)$ and embeddings $h, h^{\prime}: G \longrightarrow S^{3}, \mathcal{G}_{k}(h ; G)$ and $\mathcal{G}_{k}\left(h^{\prime} ; G\right)$ are not always isomorphic. In fact there are two-component links $L_{1}$ and $L_{2}$ such that $\mathcal{G}_{3}\left(L_{1}\right) \cong \mathbb{Z} \oplus \mathbb{Z}$ and $\mathcal{G}_{3}\left(L_{2}\right) \cong \mathbb{Z} \oplus \mathbb{Z} \oplus \mathbb{Z}_{2}[\mathbf{1 6}]$.

Proof. We define a map $\phi: \mathcal{G}_{k}(O) \longrightarrow \mathcal{G}_{k}(K)$ by

$$
\phi\left([F(O ; \mathcal{L}, \mathcal{B})]_{k}\right)=[K \# F(O ; \mathcal{L}, \mathcal{B})]_{k}
$$

for each $[F(O ; \mathcal{L}, \mathcal{B})]_{k} \in \mathcal{G}_{k}(O)$, where $\mathcal{L}$ is a set of links of type $k-1$ and \# means the connected sum of oriented knots. Clearly this is well-defined. By Lemma 2.2 , any band sum $F(K ; \mathcal{L}, \mathcal{B})$ of $K$ with links $\mathcal{L}$ of type $k-1$ is $A_{k^{-}}$equivalent to $K \# F\left(O ; \mathcal{L}^{\prime}, \mathcal{B}^{\prime}\right)$ for some links $\mathcal{L}^{\prime}$ of type $k-1$ and $\mathcal{B}^{\prime}$. Hence $\phi$ is surjective. For $\left[F\left(O ; \mathcal{L}_{i}, \mathcal{B}_{i}\right)\right]_{k} \in \mathcal{G}_{k}(O)(i=1,2)$, we have

$$
\begin{aligned}
& \phi\left(\left[F\left(O ; \mathcal{L}_{1}, \mathcal{B}_{1}\right)\right]_{k}+O\left[F\left(O ; \mathcal{L}_{2}, \mathcal{B}_{2}\right)\right]_{k}\right) \\
& =\phi\left(\left[F\left(O ; \mathcal{L}_{1} \cup \mathcal{L}_{2}, \mathcal{B}_{1} \cup \mathcal{B}_{2}\right)\right]_{k}\right) \\
& =\left[K \# F\left(O ; \mathcal{L}_{1} \cup \mathcal{L}_{2}, \mathcal{B}_{1} \cup \mathcal{B}_{2}\right)\right]_{k} \\
& =\left[F\left(K ; \mathcal{L}_{1} \cup \mathcal{L}_{2}, \mathcal{B}_{1} \cup \mathcal{B}_{2}\right)\right]_{k} \\
& =\left[F\left(K ; \mathcal{L}_{1}, \mathcal{B}_{1}\right)\right]_{k}+_{K}\left[F\left(K ; \mathcal{L}_{2}, \mathcal{B}_{2}\right)\right]_{k} \\
& =\left[K \# F\left(O ; \mathcal{L}_{1}, \mathcal{B}_{1}\right)\right]_{k}+_{K}\left[K \# F\left(O ; \mathcal{L}_{2}, \mathcal{B}_{2}\right)\right]_{k} \\
& =\phi\left(\left[F\left(O ; \mathcal{L}_{1}, \mathcal{B}_{1}\right)\right]_{k}\right)+_{K} \phi\left(\left[F\left(O ; \mathcal{L}_{2}, \mathcal{B}_{2}\right)\right]_{k}\right) .
\end{aligned}
$$

This implies that $\phi$ is a homomorphism. In order to complete the proof, we show that $\phi$ is injective. Suppose that $[K \# F(O ; \mathcal{L}, \mathcal{B})]_{k}=[K]_{k}$. By Lemma 3.2 , there is a knot $K^{\prime}$ such that $\left[K^{\prime} \# K\right]_{k}=[O]_{k}$. Then we have

$$
\begin{aligned}
{[F(O ; \mathcal{L}, \mathcal{B})]_{k} } & =\left[\left(K^{\prime} \# K\right) \# F(O ; \mathcal{L}, \mathcal{B})\right]_{k} \\
& =\left[K^{\prime} \#(K \# F(O ; \mathcal{L}, \mathcal{B}))\right]_{k} \\
& =\left[K^{\prime} \# K\right]_{k} \\
& =[O]_{k} .
\end{aligned}
$$


This implies that $\operatorname{ker} \phi=\left\{[O]_{k}\right\}$.

Habiro originated 'clasper theory' and showed Lemma 3.2 for $C_{k}$-moves [3] and [4]. The following proof is a translation of his proof in terms of band sum description of knots:

Lemma 3.2. For any knot $K$ and any integer $k \geq 1$, there is a knot $K^{\prime}$ such that $K^{\prime} \# K$ is $A_{k}$-equivalent to a trivial knot.

Proof. We shall prove this by induction on $k$. The case $k=1$ is clear. Suppose that there is a knot $K^{\prime}$ such that $K^{\prime} \# K$ is $A_{k-1}$-equivalent to a trivial knot $O(k>1)$. By Proposition 2.1, we may assume that $O=$ $F\left(K^{\prime} \# K ; \mathcal{L}, \mathcal{B}\right)$, where $\mathcal{L}$ is a set of links of type $k-1$. Then, by Lemma 2.2 , we see that $F\left(K^{\prime} \# K ; \mathcal{L}, \mathcal{B}\right)$ is $A_{k^{-}}$-equivalent to some $K \# F\left(K^{\prime} ; \mathcal{L}, \mathcal{B}^{\prime}\right)$. This completes the proof.

Let $\mathcal{K}_{k}$ be the set of $A_{k}$-equivalence classes of all oriented knots. For $[K]_{k},\left[K^{\prime}\right]_{k} \in \mathcal{K}_{k}$, we define $[K]_{k}+\left[K^{\prime}\right]_{k}=\left[K \# K^{\prime}\right]_{k}$. Then the following, shown by Habiro [3],[4] in the case that $A_{k}$-moves coincide with $C_{k}$-moves, is an immediate consequence of Lemma 3.2.

Theorem 3.3. The set $\mathcal{K}_{k}$ forms an abelian group under ' + ' with the unit element $[O]_{k}$, where $O$ is a trivial knot.

\section{Generalized $A_{k}$-move.}

In this section, we define a generalized $A_{k}$-move. For this move, several results similar to that in Sections 1, 2 and 3 hold.

Let $T$ and $S$ be trivial tangles such that $(T, S)$ and $(S, T)$ are equivalent. Let $t_{1}, t_{2}, \ldots, t_{n}$ and $s_{1}, s_{2}, \ldots, s_{n}$ be the components of $T$ and $S$ respectively. An $A_{1}(T, S)$-move is this local move $(T, S)$. Suppose that $A_{k}(T, S)$-moves are defined. For each $A_{k}(T, S)$-move $\left(T_{k}, S_{k}\right)$ we choose a trivialization of $T_{k}$ and fix it. Then the band trivializations of $\left(T_{k}, S_{k}\right)$ with respect to the trivialization are called $A_{k+1}(T, S)$-moves. Let $\left(T_{k}, S_{k}\right)$ be an $A_{k}(T, S)$-move and $D_{1}, D_{2}, \ldots, D_{n+k-1}$ the fixed trivialization of $T_{k}=t_{1} \cup t_{2} \cup \cdots \cup t_{n+k-1}$. We set $\alpha=\partial B^{3} \cap\left(D_{1} \cup D_{2} \cup \cdots \cup D_{n+k-1}\right)$ and $\beta=S_{k}$. A link $L$ in $S^{3}$ is called type $(k ;(T, S))$ if there is an orientation preserving embedding $\varphi: B^{3} \longrightarrow S^{3}$ such that $L=\varphi(\alpha \cup \beta)$. Then the pair $(\alpha, \beta)$ is called a link model of $L$. As in Section $1, A_{k}(T, S)$-move gives an equivalence relation, $A_{k}(T, S)$-equivalence, on the set of all embeddings of $G$ into $S^{3}$. For an embedding $f: G \longrightarrow S^{3}$, let $[f]_{k}$ denote the $A_{k}(S, T)$-equivalence class of $f$. Let $h: G \longrightarrow S^{3}$ be an embedding and $H$ an abelian group. Let $\varphi:[h]_{k-1} \longrightarrow H$ be an invariant. We can define that $\varphi$ is a finite type invariant of order $(n ; k ;(T, S))$ as in Section 1.

By the arguments similar to that in Sections 1, 2 and 3, we have the following five theorems: 
Theorem 4.1. Let $f, g: G \longrightarrow S^{3}$ be $A_{k-1}(T, S)$-equivalent embeddings. Then they are $A_{k}(T, S)$-equivalent if and only if $\varphi(f)=\varphi(g)$ for any finite type $A_{k}(T, S)$-equivalence invariant $\varphi$ of order $(1 ; k ;(T, S))$.

Let $h: G \longrightarrow S^{3}$ be an embedding. For $\left[f_{1}\right]_{k},\left[f_{2}\right]_{k} \in[h]_{k-1} /\left(A_{k}(T, S)\right.$ equivalence), we can define $\left[f_{1}\right]_{k}+{ }_{h}\left[f_{2}\right]_{k}$ as in Section 2 , and we have:

Theorem 4.2. The set $[h]_{k-1} /\left(A_{k}(T, S)\right.$-equivalence) forms an abelian group under ' $+{ }_{h}$ ' with the unit element $[h]_{k}$.

We denote this group by $\mathcal{G}_{k(T, S)}(h ; G)$ and call it the $A_{k}(T, S)$-equivalence group of the spatial embeddings of $G$ with the unit element $[h]_{k}$.

Theorem 4.3. Let $h_{1}, h_{2}: G \longrightarrow S^{3}$ be $A_{k-1}(T, S)$-equivalent embeddings. Then the groups $\mathcal{G}_{k(T, S)}\left(h_{1} ; G\right)$ and $\mathcal{G}_{k(T, S)}\left(h_{2} ; G\right)$ are isomorphic.

For an embedding $h: S^{1} \longrightarrow S^{3}$, let $K=h\left(S^{1}\right)$ and let $\mathcal{G}_{k(T, S)}(K)$ denote the $A_{k}(T, S)$-equivalence group $\mathcal{G}_{k(T, S)}\left(h ; S^{1}\right)$ with the unit element $[h]_{k}$.

Theorem 4.4. Let $O$ be a trivial knot. If any two knots are $A_{1}(T, S)$ equivalent, then for any oriented knot $K, \mathcal{G}_{k(T, S)}(O)$ and $\mathcal{G}_{k(T, S)}(K)$ are isomorphic.

Let $\mathcal{K}_{k(T, S)}$ be the set of $A_{k}(T, S)$-equivalence classes of all oriented knots. For $[K]_{k},\left[K^{\prime}\right]_{k} \in \mathcal{K}_{k(T, S)}$, we define $[K]_{k}+\left[K^{\prime}\right]_{k}=\left[K \# K^{\prime}\right]_{k}$.

Theorem 4.5. If any two knots are $A_{1}(T, S)$-equivalent, then the set $\mathcal{K}_{k(T, S)}$ forms an abelian group under ' + ' with the unit element $[O]_{k}$, where $O$ is a trivial knot.

Remark. If $(T, S)$ is the \#-move defined by Murakami [6], then $\mathcal{K}_{k(T, S)}$ is an abelian group.

\section{5. $A_{k}$-moves and Vassiliev invariants.}

Let $G$ be a finite graph. We give and fix orientations of the edges of $G$. Let $\mathcal{E}$ be the set of the ambient isotopy classes of the embeddings of $G$ into $S^{3}$. Let $\mathbb{Z} \mathcal{E}$ be the free abelian group generated by the elements of $\mathcal{E}$. A crossing vertex is a double point of a map from $G$ to $S^{3}$ as in Figure 5.1. An $i$ singular embedding is a map from $G$ to $S^{3}$ whose multiple points are exactly $i$ crossing vertices. By the formula in Figure 5.2 we identify an $i$-singular embedding with an element in $\mathbb{Z} \mathcal{E}$. Let $\mathcal{R}_{i}$ be the subgroup of $\mathbb{Z} \mathcal{E}$ generated by all $i$-singular embeddings. Note that $\mathcal{R}_{i}$ is independent of the choices of the edge orientations. Let $H$ be an abelian group. Let $\varphi: \mathcal{E} \longrightarrow H$ be a map. Let $\widetilde{\varphi}: \mathbb{Z} \mathcal{E} \longrightarrow H$ be the natural extension of $\varphi$. We say that $\varphi$ is a Vassiliev invariant of order $n$ if $\widetilde{\varphi}\left(\mathcal{R}_{n+1}\right)=\{0\}$. Let $\iota: \mathcal{E} \longrightarrow \mathbb{Z} \mathcal{E}$ be the natural inclusion map and $\pi_{i}: \mathbb{Z} \mathcal{E} \longrightarrow \mathbb{Z} \mathcal{E} / \mathcal{R}_{i}$ the quotient homomorphism. 
Let $u_{i-1}=\pi_{i} \circ \iota: \mathcal{E} \longrightarrow \mathbb{Z} \mathcal{E} / \mathcal{R}_{i}$ be the composition map. Then $\varphi$ is a Vassiliev invariant of order $n$ if and only if there is a homomorphism $\hat{\varphi}: \mathbb{Z} \mathcal{E} / \mathcal{R}_{n+1} \longrightarrow H$ such that $\varphi=\hat{\varphi} \circ u_{n}$. In the following we sometimes do not distinguish between an embedding and its ambient isotopy class so long as no confusion occurs.

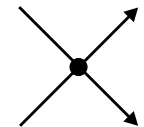

Figure 5.1.
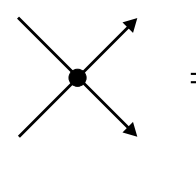

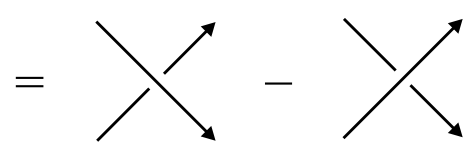

Figure 5.2.

Theorem 5.1. Let $f, g: G \longrightarrow S^{3}$ be $A_{k+1}$-equivalent embeddings. Then $u_{k}(f)=u_{k}(g)$.

By using induction on $k$, we see that an $A_{k}$-move $(T, S)$ is a $(k+1)$ component Brunnian local move, i.e., $T-t$ and $S-s$ are ambient isotopic in $B^{3}$ relative $\partial B^{3}$ for any $t \in T$ and $s \in S$ with $\partial t=\partial s$ [15]. It is not hard to see that if two embeddings $f$ and $g$ are related by a $(k+1)$-component Brunnian local move, then $f$ and $g$ are $k$-similar, where $k$-similar is an equivalence relation defined by the first author [13]. Therefore, we note that Theorem 5.1 follows from [1] or [9]. However we give a self-contained proof here.

Let $T$ be a tangle. Let $\mathcal{H}(T)$ be the set of all (possibly nontrivial) tangles that are homotopic to $T$ relative to $\partial B^{3}$. Let $\mathcal{E}(T)$ be the quotient of $\mathcal{H}(T)$ by the ambient isotopy relative to $\partial B^{3}$. Then $\mathbb{Z} \mathcal{E}(T), i$-singular tangles and $\mathcal{R}_{i}(T) \subset \mathbb{Z} \mathcal{E}(T)$ are defined as above.

Proof. It is sufficient to show for each $A_{k}$-move $(T, S)$ that $T-S$ is an element in $\mathcal{R}_{k}(T)$. We show this by induction on $k$. The case $k=1$ is clear. Recall that an $A_{k}$-move $(T, S)$ is a band trivialization of an $A_{k-1}$-move, say $\left(T^{\prime}, S^{\prime}\right)$. Then we have that $T-S=X_{1}-X_{2}$ where $X_{1}$ and $X_{2}$ are 1-singular tangles in Figure 5.3. Let $Y_{1}$ and $Y_{2}$ be 1-singular tangles in Figure 5.4. It is clear that $Y_{1}-Y_{2}=0$. Thus we have $T-S=\left(X_{1}-Y_{1}\right)-\left(X_{2}-Y_{2}\right)$. By the induction hypothesis we see that $S^{\prime}-T^{\prime}$ is an element of $\mathcal{R}_{k-1}\left(S^{\prime}\right)$. Therefore both $X_{1}-Y_{1}$ and $X_{2}-Y_{2}$ are elements of $\mathcal{R}_{k}(T)$.

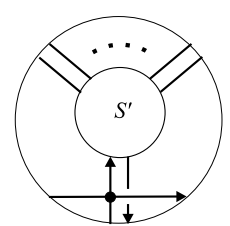

$X_{1}$

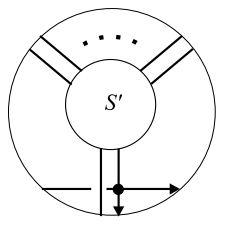

$X_{2}$

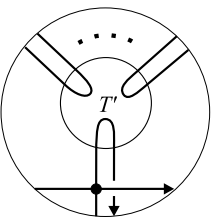

$Y_{1}$

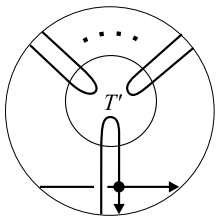

$Y_{2}$

Figure 5.3.

Figure 5.4. 
Proof of Proposition 1.3. It is sufficient to show that

$$
\sum_{X \subset\{1,2, \ldots, n+1\}}(-1)^{|X|} F\left(f ; \bigcup_{i \in X}\left\{L_{i}\right\}, \bigcup_{i \in X}\left\{B_{i}\right\}\right)
$$

is an element of $\mathcal{R}_{(n+1)(k-1)}$. We show this together with some additional claims by induction on $n$. First consider the case $n=0$. Then we have by Theorem 5.1 and its proof that $F(f ; \emptyset, \emptyset)-F\left(f ;\left\{L_{1}\right\},\left\{B_{1}\right\}\right)$ is a sum of $(k-1)$-singular embeddings each of which has all crossing vertices in the associated ball. Note that these $(k-1)$-singular embeddings are natural extensions of the $(k-1)$-singular tangles that express the difference of the $A_{k-1}$-move, and these $(k-1)$-singular tangles depends only on the link $L_{1}$. Next we consider the general case. Note that

$$
\begin{aligned}
& \sum_{X \subset\{1,2, \ldots, n+1\}}(-1)^{|X|} F\left(f ; \bigcup_{i \in X}\left\{L_{i}\right\}, \bigcup_{i \in X}\left\{B_{i}\right\}\right) \\
= & \sum_{X \subset\{1,2, \ldots, n\}}(-1)^{|X|} F\left(f ; \bigcup_{i \in X}\left\{L_{i}\right\}, \bigcup_{i \in X}\left\{B_{i}\right\}\right) \\
& -\sum_{X \subset\{1,2, \ldots, n\}}(-1)^{|X|} F\left(f ; \bigcup_{i \in X}\left\{L_{i}\right\} \cup\left\{L_{n+1}\right\}, \bigcup_{i \in X}\left\{B_{i}\right\} \cup\left\{B_{n+1}\right\}\right) .
\end{aligned}
$$

By the hypothesis we have both

$$
\sum_{X \subset\{1,2, \ldots, n\}}(-1)^{|X|} F\left(f ; \bigcup_{i \in X}\left\{L_{i}\right\}, \bigcup_{i \in X}\left\{B_{i}\right\}\right)
$$

and

$$
\sum_{X \subset\{1,2, \ldots, n\}}(-1)^{|X|} F\left(f ; \bigcup_{i \in X}\left\{L_{i}\right\} \cup\left\{L_{n+1}\right\}, \bigcup_{i \in X}\left\{B_{i}\right\} \cup\left\{B_{n+1}\right\}\right)
$$

are sums of $n(k-1)$-singular embeddings and they differ only by the band sum of $L_{n+1}$. Therefore we have

$$
\sum_{X \subset\{1,2, \ldots, n+1\}}(-1)^{|X|} F\left(f ; \bigcup_{i \in X}\left\{L_{i}\right\}, \bigcup_{i \in X}\left\{B_{i}\right\}\right)
$$

is a sum of $(n(k-1)+(k-1))$-singular embeddings. 


\section{References}

[1] M. Gusarov, On n-equivalence of knots and invariants of finite degree, in 'Topology of Manifolds and Varieties' (O. Viro, ed.), American Mathematical Society, Providence, RI, 173-192, 1994, MR 96i:57005, Zbl 0865.57007.

[2] K. Habiro, Aru musubime no kyokusyo henkei no zoku ni tuite (Japanese), Master's Thesis, University of Tokyo, 1994.

[3] _ Claspers and the Vassiliev skein modules, Ph.D. Thesis, University of Tokyo, 1997.

[4] _ Claspers and finite type invariants of links, Geom. Topol., 4 (2000), 1-83, MR 2001g:57020, Zbl 0941.57015.

[5] T. Motohashi and K. Taniyama, Delta unknotting operation and vertex homotopy of spatial graphs, in 'Proceedings of Knots '96 Tokyo' (S. Suzuki, ed.), 185-200, World Scientific Publ. Co., 1997, MR 99i:57021, Zbl 0960.57003.

[6] H. Murakami, Some metrics of classical knots, Math. Ann., 270 (1985), 35-45, MR 86g:57007, Zbl 0535.57005.

[7] _ Vassiliev invariants of type two for a link, Proc. Amer. Math. Soc., 124 (1996), 3889-3896, MR 97b:57007, Zbl 0874.57004.

[8] H. Murakami and Y. Nakanishi, On a certain move generating link-homology, Math. Ann., 284 (1989), 75-89, MR 90f:57007, Zbl 0646.57005.

[9] Y. Ohyama, Vassiliev invariants and similarity of knots, Proc. Amer. Math. Soc., 123 (1995), 287-291, MR 95f:57018, Zbl 0847.57012.

[10] Y. Ohyama, K. Taniyama and S. Yamada, Vassiliev invariants of unknotting number one knots, Tokyo J. Math., 25(1) (2002), 17-31, MR 2003d:57032, Zbl 1002.57030.

[11] T. Stanford, Finite-type invariants of knots, links and graphs, Topology, 35 (1996), 1027-1050, MR 97i:57009, Zbl 0863.57005.

[12] S. Suzuki, Local knots of 2-spheres in 4-manifolds, Proc. Japan Acad., 45 (1969), 34-38, MR 40 \#2101, Zbl 0176.53601.

[13] K. Taniyama, On similarity of links, Gakujutu Kenku (issued by School of Education, Waseda University), 14 (1992), 33-36.

[14] _ Homology classification of spatial embeddings of a graph, Topology Appl., 65 (1995), 205-228, MR 97c:57003, Zbl 0843.57012.

[15] K. Taniyama and A. Yasuhara, Realization of knots and links in a spatial graph, Topology Appl., 112 (2001), 87-109, MR 2002e:57005, Zbl 0968.57001.

[16] _ Clasp-pass moves on spatial graphs, Topology Appl., 122 (2002), 501-529, CMP 1911 697, Zbl 1001.57011.

[17] B Band description of knots and Vassiliev invariants, Math. Proc. Cambridge Philos. Soc., 133 (2002), 325-343, CMP 1912405.

[18] W.T. Wu, On the isotopy of a complexes in a Euclidean space, I, Sci. Sin., 9 (1960), 21-46, MR 23 \#A1381, Zbl 0121.18102.

[19] M. Yamamoto, Knots in spatial embeddings of the complete graph on four vertices, Topology Appl., 36 (1990), 291-298, MR 91m:57007, Zbl 0721.57005.

[20] A. Yasuhara, Disk/band surface and spatial-graph homology, Topology Appl., 69 (1996), 173-191, MR 97b:57003, Zbl 0860.57004. 
[21] _ Delta-unknotting operation and adaptability of certain graphs, in 'Proceedings of Knots '96 Tokyo' (S. Suzuki, ed.), 115-121, World Scientific Publ. Co., 1997, MR 99j:57004, Zbl 0960.57004.

Received July 11, 2000 and revised July 30, 2001.

Department of Mathematics

WASEDA UNIVERSITRY

SHINJUKU-KU

TOKYO 169-8050

JAPAN

E-mail address: taniyama@waseda.jp

Department of Mathematics

Tokyo Gakugei University

KOGANEI

TOKYO 184-8501

JAPAN

E-mail address: yasuhara@u-gakugei.ac.jp 